\title{
Nonuniversality due to inhomogeneous stress in semiconductor surface nanopatterning by low-energy ion-beam irradiation
}

\author{
A. Moreno-Barrado,,${ }^{1, *}$ M. Castro, ${ }^{2}$ R. Gago,${ }^{3}$ L. Vázquez, ${ }^{3}$ J. Muñoz-García, ${ }^{4}$ A. Redondo-Cubero, ${ }^{5}$ B. Galiana, ${ }^{6}$ \\ C. Ballesteros, ${ }^{6}$ and R. Cuerno ${ }^{4}$ \\ ${ }^{1}$ Instituto de Investigación Tecnológica (IIT) and Grupo Interdisciplinar de Sistemas Complejos (GISC), Universidad Pontificia Comillas, \\ 28015 Madrid, Spain \\ ${ }^{2}$ GISC and Grupo de Dinámica No Lineal (DNL), Escuela Técnica Superior de Ingeniería (ICAI), Universidad Pontificia Comillas, \\ 28015 Madrid, Spain \\ ${ }^{3}$ Instituto de Ciencia de Materiales de Madrid, Consejo Superior de Investigaciones Científicas, 28049 Madrid, Spain \\ ${ }^{4}$ Departamento de Matemáticas and GISC, Universidad Carlos III de Madrid, Avenida de la Universidad 30, 28911 Leganés, Spain \\ ${ }^{5}$ Departamento de Física Aplicada y Centro de Micro-Análisis de Materiales, Universidad Autónoma de Madrid, 28049 Madrid, Spain \\ ${ }^{6}$ Departamento de Física, Universidad Carlos III de Madrid, Avenida de la Universidad 30, 28911 Leganés, Spain
}

(Received 28 May 2014; revised manuscript received 20 March 2015; published 13 April 2015)

\begin{abstract}
A lack of universality with respect to ion species has been recently established in nanostructuring of semiconductor surfaces by low-energy ion-beam bombardment. This variability affects basic properties of the pattern formation process, like the critical incidence angle for pattern formation, and has remained unaccounted for. Here, we show that nonuniform generation of stress across the damaged amorphous layer induced by the irradiation is a key factor behind the range of experimental observations, as the form of the stress field is controlled by the ion/target combination. This effect acts in synergy with the nontrivial evolution of the amorphous-crystalline interface. We reach these conclusions by contrasting a multiscale theoretical approach, which combines molecular dynamics and a continuum viscous flow model, with experiments using $\mathrm{Xe}^{+}$and $\mathrm{Ar}^{+}$ ions on a $\mathrm{Si}(100)$ target. Our general approach can apply to a variety of semiconductor systems and conditions.
\end{abstract}

DOI: 10.1103/PhysRevB.91.155303

PACS number(s): 81.16.Rf, 79.20.Rf, 68.35.Ct, 05.45.-a

\section{INTRODUCTION}

Mechanical stress has been recognized, and even exploited, as a main actor in pattern-forming systems. From nanostructures [1-3] to macroscopic sandy dunes [4,5], many patterns emerge from the interplay between driving-induced stress and competing mechanisms. For swift-ion irradiation of solid targets, plastic deformations due to thermal spikes [6] are known to produce surface features measuring tens of micrometers. However, for low- to medium-energy ion-beam sputtering (IBS) of monoelemental semiconductor targets, in which pattern features conspicuously reduce to a few nanometers [7-9], the relevance of stress has been highlighted only recently $[10,11]$.

In most of these examples, the time scales for pattern formation are set by the external driving. However, in surface erosion by IBS, events induced by the ion beam, like relaxation of collision cascades, last a few picoseconds [12], while the patterns emerge on time scales of seconds [8]. Despite intensive inquiry into this nanostructuring technique, many basic questions remain open [13]. Their elucidation will impact materials science, due to a high potential for applications [14], and condensed matter physics at large, as multiscale systems like this [15] defy our current capabilities to understand their collective behavior [16].

In particular, low-energy IBS of silicon has become a paradigmatic system, due to the importance of this material for technological applications [17]. Its pattern-forming properties are widely regarded as representative of targets that are, or

*Corresponding author: ana.moreno@iit.upcomillas.es become, amorphous under this type of irradiation, like semiconductors [12]. Thus work on Si has recently highlighted [11] substantial differences between the experimental (ripple) pattern formation process at nonglancing ion incidence angles $\theta$ and the classic Bradley-Harper (BH) mechanism [18] based on the interplay between a curvature-dependent sputtering yield and thermal surface diffusion. While the BH theory predicts ripple formation for any value of $\theta$, experiments with $\mathrm{Ar}^{+}$ions [11,19-21] have determined a critical value for the incidence angle, $\theta_{c} \simeq 45^{\circ}$, below which the surface remains flat, and above which ripples emerge. Akin to continuous phase transitions, the morphological transition at $\theta=\theta_{c}$ is type II [22], since the typical length-scale, namely, the ripple wavelength $\lambda$, diverges as

$$
\lambda \sim\left|\theta-\theta_{c}\right|^{-n}
$$

with a critical exponent value $n=1 / 2$. Remarkably, for IBS in presence of metallic impurities, or of compound targets, the role of phases seems somehow reversed, since patterns form for small angles starting at $\theta=0$, see, e.g., Refs. [9,23,24], and other therein.

These crucial features are driving recent improvements over BH's theory through more sophisticated approaches, like crater functions [15,25] or hydrodynamic frameworks [10,26,27], which reproduce the transition at $\theta_{c}=45^{\circ}$ and suggest this value to be energy- and material independent. Thus experimental reports on $\theta_{c}$ values which differ from this result question the physical assumptions behind such models. For example, experiments on $\mathrm{Si}$ lead to $\theta_{c} \simeq 60^{\circ}$ for ions heavier than $\mathrm{Ar}^{+}$, like $\mathrm{Xe}^{+}$[28], or $\mathrm{Kr}^{+}$[29], or to $\theta_{c} \simeq 62^{\circ}$ for Ge bombarded with $\mathrm{Kr}^{+}[30,31]$. In analogy with a critical temperature, the value of $\theta_{c}$ should not be expected to be universal, in contrast 
TABLE I. Experimental critical angles for Si IBS with different ions and energies. For all cases $\theta_{c} \in\left[45^{\circ}, 70^{\circ}\right]$, consistent with the predictions of Eq. (25) for a non-BH scenario, as supported by MD. Experiments on Ge are given for reference, suggesting generality of results for amorphizable materials.

\begin{tabular}{lcc}
\hline \hline System & $\theta_{c}$ & Reference \\
\hline $\mathrm{Ar}^{+} \rightarrow \mathrm{Si}(250 \mathrm{eV}-1 \mathrm{keV})$ & $48^{\circ}$ & {$[20,21]$} \\
$\mathrm{Ar}^{+} \rightarrow \mathrm{Si}(300 \mathrm{eV}-1.1 \mathrm{keV})$ & $45^{\circ}-50^{\circ}$ & {$[11]$} \\
$\mathrm{Kr}^{+} \rightarrow \mathrm{Si}(2 \mathrm{keV})$ & $60^{\circ}$ & {$[29]$} \\
$\mathrm{Ar}^{+} \rightarrow \mathrm{Si}(500 \mathrm{eV}-1 \mathrm{keV})$ & $46^{\circ}$ & {$[11] ;$ this work } \\
$\mathrm{Xe}^{+} \rightarrow \mathrm{Si}(500 \mathrm{eV}-1 \mathrm{keV})$ & $58^{\circ}$ & this work \\
$\mathrm{Kr}^{+} \rightarrow \mathrm{Ge}(1 \mathrm{keV})$ & $62^{\circ}$ & {$[30]$} \\
$\mathrm{Kr}^{+} \rightarrow \mathrm{Ge}(1.2 \mathrm{keV})$ & $\leqslant 65^{\circ}$ & {$[31]$} \\
$\mathrm{Xe}^{+} \rightarrow \mathrm{Ge}(1.2 \mathrm{keV})$ & $\leqslant 70^{\circ}$ & {$[31]$} \\
\hline \hline
\end{tabular}

with critical exponents like $n$ in Eq. (1). Table I summarizes these and other experimental results.

In this paper, we elucidate the ion-induced stress field that builds up throughout the damaged amorphous layer evolving on top of the solid target, as the key physical effect driving pattern formation in low-energy IBS of semiconductor surfaces. In line with recent work in the context of surface nanostructuring of IBS (see a recent review in Ref. [17]), we focus in the case of silicon targets, which is widely believed to be important for potential applications, while being representative of semiconductor targets. Within the current revision of the basic mechanisms for pattern formation that improve upon the classic BH view [17], additional important classes of materials, like metals, are expected to differ substantially. Metals do not become amorphous under similar conditions [8,32], their pattern formation processes being possibly controlled by different mechanisms of material transport.

For the case of silicon targets, we specifically show that it is the nontrivial space distribution of ion-induced stress, as a function of the ion/target combination, which is responsible for the variety of experimental observations. This is a promising insight into the physical mechanism that controls similarities and differences in ripple formation by IBS for different semiconductors. We reach these conclusions via a multiscale description of the $\mathrm{Si}$ system by molecular dynamics (MD) simulations, combined with a generalization of the stressdriven viscous flow model in Refs. [10,11], both of which are contrasted with experiments with $\mathrm{Xe}^{+}$and $\mathrm{Ar}^{+}$ions.

\section{METHODS}

\section{A. Overview of the methodology used in this work}

One of the goals of our work is to integrate different approaches, from experimental to theoretical, passing through molecular dynamics simulations. In order to illustrate the complexity of such an integration, Fig. 1 provides a graphical summary of the methods we have employed and how they are put together to provide testable predictions that allow to validate the theory against experiments.

Given the insufficiency of $\mathrm{BH}$ theory to predict nonzero values of the critical angle $\theta_{c}$, we need to resort to alternative continuum descriptions. For instance, the so-called crater

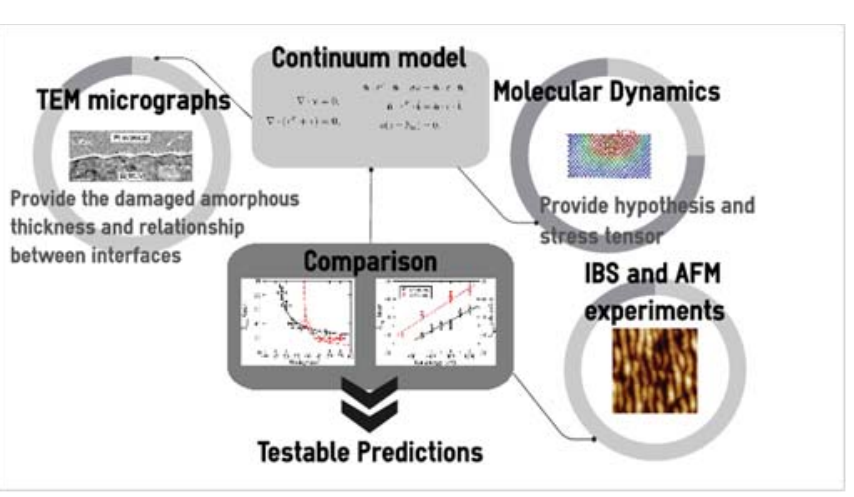

FIG. 1. (Color online) Schematic summary of the methods and approaches employed in this work.

functions replace the space distribution of energy deposition employed in the BH model by a kernel which is directly evaluated by MD [15,25] or by binary-collision Monte Carlo [33] simulations. However, the consistency of some approaches of this class has been recently questioned [34-36], while dependencies of pattern properties with physical parameters are not predicted. An alternative continuum framework is provided by hydrodynamic models of IBS ripple formation, based on the generation of ion-induced stress [10,26,27]. Actually, these models yield predictions on, e.g., the energy dependence of the ripple wavelength, which have been validated recently [11]. However, as mentioned above basically all these models lead to $\theta_{c}=45^{\circ}$, irrespective of conditions on energy or ion/target combination. Our goal is to improve these continuum models through a more detailed implementation of results from MD simulations and/or experiments. In turn, analytical predictions will be contrasted back with experimental results. This is the combined approach, which is illustrated in Fig. 1.

\section{B. Sample preparation}

Our experimental data are obtained from commercial $p$ type $\mathrm{Si}(100)$ targets $(380-\mu \mathrm{m}$ thick, $1-10 \mathrm{~m} \Omega \mathrm{cm})$, which have been sputtered with $\mathrm{Xe}^{+}$and $\mathrm{Ar}^{+}$beams for different incidence angles within the 300-1100 eV energy range, as in Ref. [11]. The ions were extracted from a commercial Kaufman source [Veeco(c)] with a 3-cm grid system. Particular care has been taken to avoid metal contamination, which has been checked by Rutherford backscattering spectrometry. The angle of incidence has an overall resolution of $\pm 2^{\circ}$. The current density at the sample position in the plane parallel to the source grids $\left(J_{0}\right)$ was set in the $30-50 \mu \mathrm{A} / \mathrm{cm}^{2}$ range. Low $J_{0}$ values were aimed at assessing the early-stage linear regime, following the criteria reported in Ref. [11].

\section{Sample analysis}

The surface morphology was imaged $e x$-situ with an AFM Nanoscope IIIa equipment (Bruker) operating in intermittentcontact mode, using Si cantilevers (Bruker) with nominal radius of curvature of $8 \mathrm{~nm}$. A sample topography is shown in Fig. 2(a). We have also employed high-resolution transmission electron microscopy (HRTEM) for microstructural analysis of the ripple features. Special care has been taken during the specimen preparation for HRTEM observations in order 


\section{(b)}

(a)

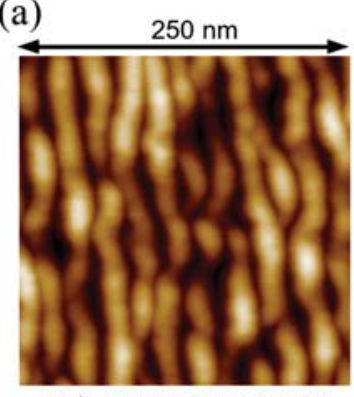

$\mathrm{Xe}+\mathrm{Si}\left(\theta=65^{\circ}, \mathrm{E}=500 \mathrm{eV}\right)$

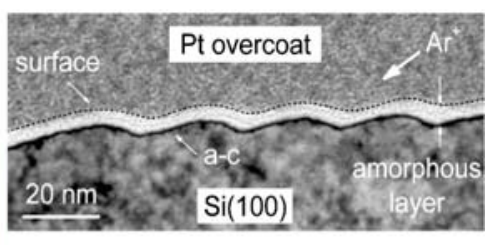

(c)

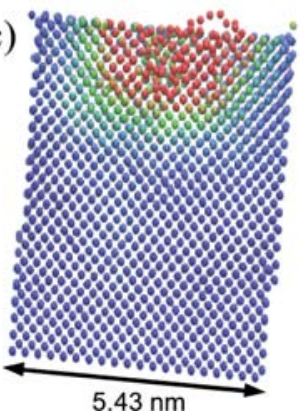

FIG. 2. (Color online) (a) AFM top view of experimental ripples obtained by $\mathrm{Xe}^{+}$IBS of Si for $E=500 \mathrm{eV}$ and $\theta=65^{\circ}$. (b) HRTEM cross-sectional high-resolution image along the $\langle 110\rangle$ direction. The free interface is marked by dots. (c) MD simulation after one $\mathrm{Xe}^{+}$ impact under the same conditions as in (a). Different colors stand for different values of the temperature, which decreases with distance from the ion entry.

not to modify the original Si-amorphous surface layer that develops under low-energy irradiation. Regarding this point, lamellae for electron transparency were prepared using a focused ion beam (FIB), protecting the sample surface through pre-deposition of a Pt layer, and milling with $\mathrm{Ga}^{+}$ions close to normal incidence. Samples are examined in a Philips Tecnai F20 operated at $200 \mathrm{keV}$. An example is shown in Fig. 2(b). The focus and contrast conditions of the multibeam image are selected to magnify the amorphous crystalline interface contrast. Note that, akin to experiments at medium energies [37], the free and the amorphous-crystalline (a-c) interfaces that bound the amorphous layer both exhibit similarly rippled topographies with a horizontal shift, motivating hypothesis to be made in our continuum model below.

The contrast variations observed in crystalline $\mathrm{Si}$ in the low magnification image are associated with small thickness variations and damage, induced through FIB besides the cross-section surface. In order to reduce this damage in semiconductor samples prepared for TEM, commonly postFIB $\mathrm{Ar}^{+}$ion milling is employed at an incidence grazing angle. However, in this work, no FIB post-treatments were employed, so that the irradiated sample surface could be protected. The size of the Si amorphous layer (which appears brighter in the image), the amorphous-crystalline interface, and the Pt-deposited layer are analyzed using HRTEM images along the $\langle 110\rangle$ direction. An example is shown in the bottom larger image of Fig. 3, where the thickness variation of the amorphous layer associated with the topography is clearly visible, ranging from 3 to $6 \mathrm{~nm}$.

\section{Molecular dynamics simulations}

Collision cascades induce permanent displacement of target atoms and damage $[8,9,12]$. Sustained irradiation creates

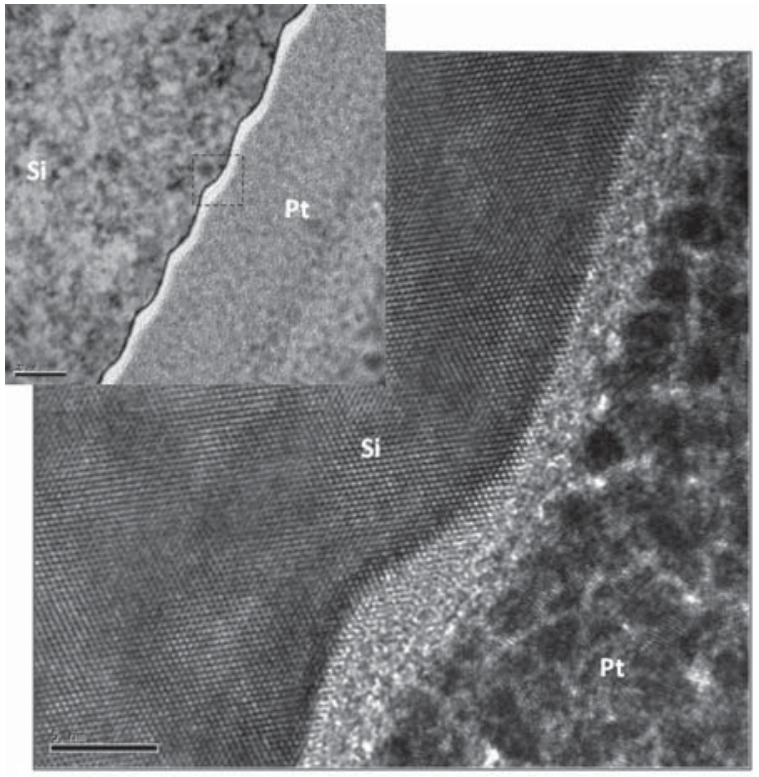

FIG. 3. (Small, upper image) Cross-sectional multibeam image along the $\langle 110\rangle$ direction, corresponding to Fig. 2(a). The bar corresponds to $20 \mathrm{~nm}$. (Large, lower image) Corresponding HRTEM image of the area marked with a square in the center of the upper image. The HRTEM image allows to appreciate the characteristic $\{111\}$ Si-lattice fringes of the crystalline phase. Thickness variations in the amorphous-Si layer are also visible, together with the Pt layer. The bar corresponds to $5 \mathrm{~nm}$.

a well-defined amorphous layer on top of a crystalline bulk [11,38-40]. The residual stress thus built up throughout the layer is partially relaxed by defect motion and sputtering $[41,42]$, and is described by a stress tensor $\tau$. Thus microscopic defect creation produces macroscopic stress. As an illustration, the amorphous layer that develops on Si under oblique incidence pattern forming conditions as in the ripple structure of Fig. 2(a) can be clearly identified in a HRTEM cross-sectional view like the one provided by Fig. 2(b). In Fig. 2(c), we show a representative view of the simulated (microscopic) target after a single ion impact, for ion energy $E$ and incidence angle $\theta$ as in Fig. 2(a). Accumulation of such damage will eventually lead to an amorphized layer like the one produced in the experiments.

In order to characterize the stress distribution generated under irradiation, we have performed MD simulations using the LAMMPS software [43] as follows. A crystalline $4.89 \times 4.89 \times 10.83 \mathrm{~nm}^{3}$ Si target is bombarded with $\mathrm{Xe}^{+}$at $300 \mathrm{eV}-1 \mathrm{keV} \mathrm{Xe}^{+}$ions. The interaction potential used in this work is Tersoff, interpolated with a Ziegler-Biersack-Littmark potential [44-47]. As the collision cascade is around two unit cells width ( $\simeq 1 \mathrm{~nm}$ ), we have used a system size large enough to avoid that the periodicity of the boundaries (where the thermostats are located) affects the computation of stress. We have reproduced one of the simulations by doubling the lateral size and, within the error bars, the results (not shown) are unchanged. Besides this, we compute the stress only for a subset of the atoms that are far apart from those boundaries.

Each simulation corresponds to 1000 consecutive impacts, allowing to describe the build-up of stress inside the target. 

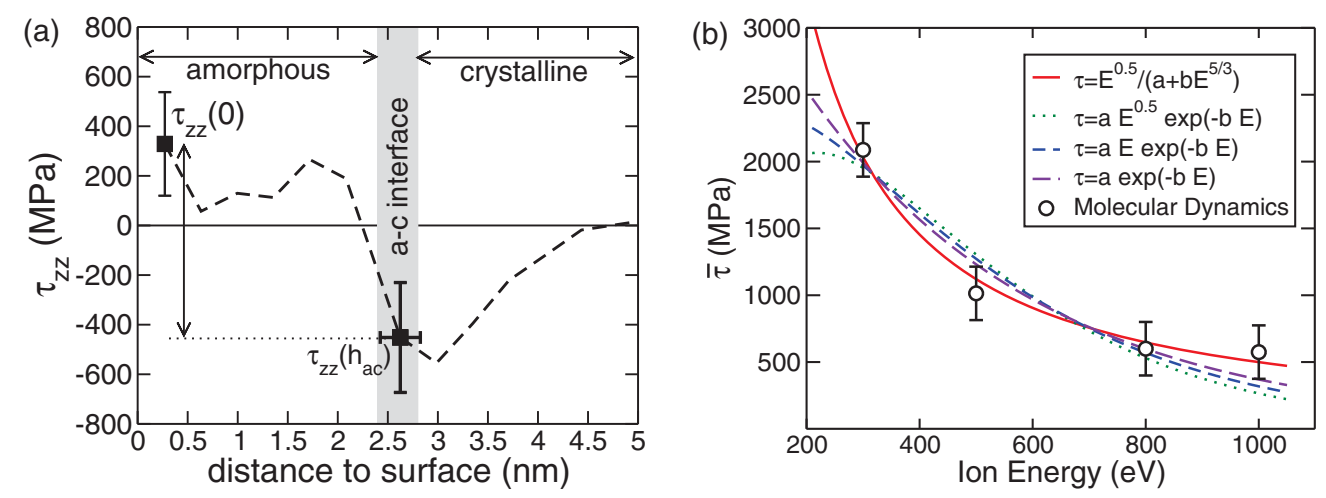

FIG. 4. (Color online) MD simulations of $\mathrm{Xe}^{+} \rightarrow \mathrm{Si}$. (a) Diagonal element of the excess (ion-induced) stress tensor in the normal direction, $\tau_{z z}$, for $E=300 \mathrm{eV}$. Note the nonuniform distribution of stress across the amorphous layer. (b) Dependence of the total stress $\bar{\tau}$ with ion energy (circles are simulation data). In this range of energies, the numerical results are compatible with different models for defect relaxation [48], as detailed in the legend (nonsolid lines). Simple Davis' scaling [41] (solid line) seems to capture the simulation results better.

This contrasts with most of the standard MD approaches in which averaging is performed over different impacts on the same pristine material. All the presented simulations were performed using normal incidence A simulation was performed at $\theta=60^{\circ}$ to confirm that the stress tensor at oblique ion incidence can be obtained (within numerical error) as a tensorial rotation of that at $0^{\circ}$.

Between impacts, the system is relaxed so that the impactinduced mechanical oscillations are damped out $(\simeq 10 \mathrm{ps})$ and the temperature is rescaled to $300 \mathrm{~K}$ in order to preserve the experimental, almost isothermal, initial conditions for the next impact. Also, in order to reduce computation time, we have employed an adaptive time-step with a positive feedback when particles start to move slowly. Besides, sputtered atoms were removed from the system.

In order to simulate an infinite solid, periodic boundary conditions have been considered in the $X-Y$ directions while free surfaces have been set perpendicular to the $Z$ axis. We surround the central part of the system with one-unit-cell layer of silicon, with a velocity viscous damping in order to remove sound waves that create artificial oscillations in the stress tensor. We used an NVE ensemble that allows temperature and pressure to react and adapt to the momentum and energy exchange between every incident ion and the target material.

The location of the amorphous-crystalline interface (a-c) is subtle as one has to define a surface from a collection of atoms. We have used three alternative ways to compute it that result into the horizontal error bar (of around $0.5 \mathrm{~nm}$ ) in the shaded area in Fig. 4(a). One is to interpolate between the density of the crystalline (large $z$ ) and damaged regions (averaged close to the surface) and perform a Maxwell construction between them. Alternatively, either by computing the average coordination number for every atom and or performing a similar calculation as with the density but for the $z z$ component of the stress tensor. For the three observables (density, coordination number or stress), it is simpler to work with the cumulative integral of these observables as it reduces numerical noise. In all cases, the location of the a-c interface is similar.

Finally, the stress tensor is computed without the virial term arising from kinetic energy contributions, see Ref. [43] for details. Throughout our present work we consider negative (positive) total stress to be compressive (tensile).

\section{THEORY}

\section{A. Hypothesis extracted from molecular dynamics simulations}

Our MD simulations allow us to check quantitatively previous assumptions $[10,11]$ on the stress distribution generated under IBS. In particular, the analysis of the simulations imply the following. (i) The ion-induced stress tensor $\tau$ has a negligible trace, with the components perpendicular to the ion direction being equal; hence, overall the damaged amorphous layer can be assumed to be an incompressible fluid. (ii) As postulated in Ref. [10], the value of $\tau$ at an arbitrary incidence angle $\theta$ is approximated well by the rotation of the tensor obtained under normal ion incidence $(\theta=0)$. (iii) Beyond earlier simplifications $[10,11]$, the $\tau_{i j}$ components depend nontrivially on the distance to the free surface, see $\tau_{z z}$ in Fig. 4(a). In particular, the ion-induced stress takes different values at the free interface and within the bulk of the amorphous layer. (iv) The energy dependence of the total stress $\bar{\tau}=\operatorname{Trace}\left(\tau+\tau^{F}\right)$, where $\tau^{F}$ is the standard fluidic contribution to stress [see Eq. (4) below], follows closely Davis' scaling [41], being insensitive to details in the stress relaxation mechanism by defect motion. Namely, different alternatives for the functional dependence of total stress with ion energy [48] are also compatible with our simulation data in the range of energies considered, see Fig. 4(b), although the simpler Davis' scaling seems to suffice, as experimentally verified recently [11]. Although the original model formulated by Davis was based on spike formation, later results [49] showed that binary collisions, more relevant at the low energies we are considering, lead to the same scaling of stress with ion energy for $E \lesssim 1 \mathrm{keV}$. Our MD results imply that the interplay between ion-induced stress, viscous flow dynamics, and sputtering leads to partial defect relaxation, which is the basic idea of Davis' model for stress evolution in irradiated materials. We note that very recent measurements [42] of stress evolution during low-energy argon ion bombardment of Si seem to favor specifically a bimolecular recombination mechanism for individual flow defects [50]. 


\section{B. Model equations}

In spite of the relevance of the information provided by MD simulations, this computational framework cannot describe quantitatively by itself the time evolution of the ripple patterns, which takes place in time scales of seconds and minutes, and in length scales of tens of nanometers [17]. Comparison between Figs. 2(a) and 2(c) illustrates the huge scale separation between experiments and MD simulations.

In order to bridge between our MD results and the typical scales of the patterns, we formulate a hydrodynamic model for the damaged layer, assumed to be an incompressible fluid, basing the assumptions to be made on the MD results (i)-(iv) described above. Assuming further that the layer has a high (uniform) viscosity, the equations of motion read [51]

$$
\begin{aligned}
& \nabla \cdot \mathbf{v}=0, \\
& \nabla \cdot \hat{\tau}=\mathbf{0},
\end{aligned}
$$

where $\mathbf{v}$ is the velocity field in the amorphous layer and the global stress tensor, $\hat{\tau}=\tau^{F}+\tau$ in Eq. (3), is the sum of a fluidic contribution $\tau^{F}$ and the external contribution, $\tau$, that comprises the cumulative effect of the damage produced by irradiation. The mathematical expression for $\tau^{F}$ reads [51]

$$
\tau_{i j}^{F}=-p \delta_{i j}+\mu\left(\frac{\partial v_{i}}{\partial x_{j}}+\frac{\partial v_{j}}{\partial x_{i}}\right),
$$

where $p$ is the hydrostatic pressure and $\mu$ is the viscosity of the damaged amorphous layer. For simplicity, since we are mainly interested in behavior close to the critical angle for ripple formation, we will neglect the space dependence along ripple ridges and consider a two-dimensional system. Taking $x$ and $z$ to denote the coordinates along the substrate and along the growth direction, respectively, $\tau^{F}$ can be written as

$$
\tau^{F}=\left(\begin{array}{cc}
-p+2 \mu \partial_{x} u & \mu\left(\partial_{z} u+\partial_{x} w\right) \\
\mu\left(\partial_{z} u+\partial_{x} w\right) & -p+2 \mu \partial_{z} w
\end{array}\right),
$$

where $u(x, z, t)$ and $w(x, z, t)$ are, respectively, the horizontal and vertical components of the fluid velocity at time $t$.

Determining the precise local values of the components of the ion-induced stress distribution, $\tau$, would require experimental measurements and/or a highly accurate MD parametrization. In absence of such type of high-resolution data, we consider the main qualitative conclusions from our MD simulations. Thus we assume the trace of the $\tau$ tensor to be negligible, and its components to depend on the distance to the free surface. In a coordinate system $\left(x^{\prime}, z^{\prime}\right)$ in which $z^{\prime}$ is oriented along the ion beam direction, see Fig. 5, the simplest form for the ion-induced stress tensor compatible with these assumptions reads

$$
\tau^{\prime}=\tau_{z z}\left(z^{\prime}\right)\left(\begin{array}{rr}
1 & 0 \\
0 & -1
\end{array}\right)
$$

its divergence in this coordinate system reducing to $\vec{\nabla}^{\prime} \cdot \tau^{\prime}=$ $\left(0,-\partial_{z^{\prime}} \tau_{z z}\left(z^{\prime}\right)\right)$.

Coordinates in the laboratory system are related with $\left(x^{\prime}, z^{\prime}\right)$ through $(x, z)=\left(x^{\prime}, z^{\prime}\right) \cdot R(\theta)^{T}$, where $R(\theta)$ is the rotation

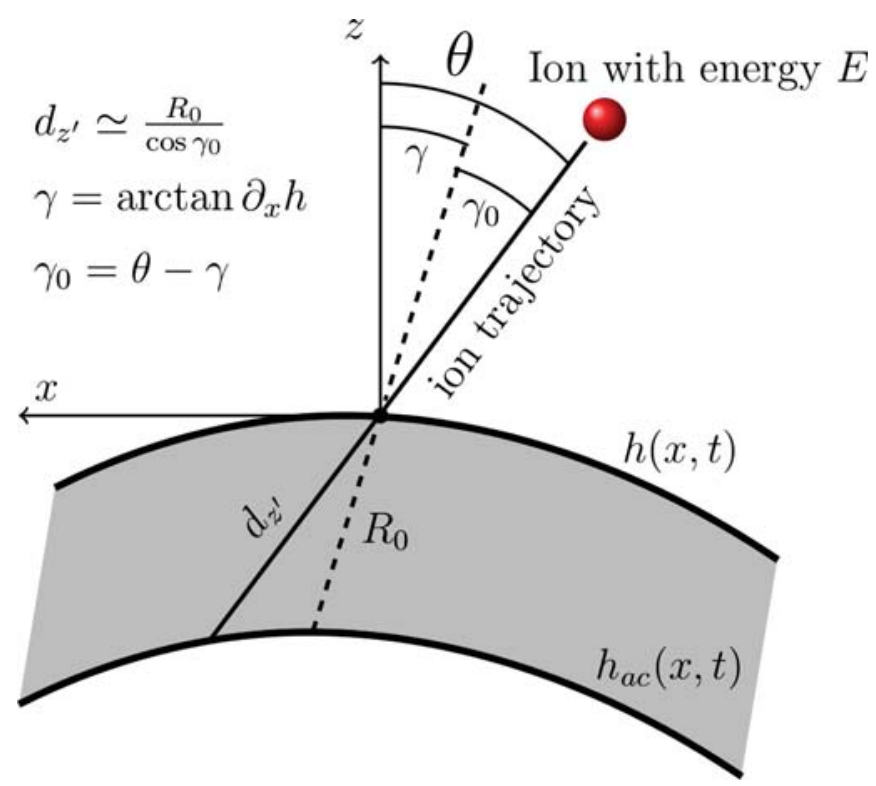

FIG. 5. (Color online) Laboratory coordinate system and geometrical quantities defined in the main text. We illustrate the geometrical relations between the distance along the ion beam trajectory, $d_{z^{\prime}}$, and the thickness of the amorphous layer, $R_{0}$; and between the laboratory and local ion incidence angles, $\theta$ and $\gamma_{0}$, respectively, and the local inclination angle, $\gamma$. The amorphous layer is represented as the grey area, being bounded by the free and the amorphous-crystalline interfaces, which are denoted by $h$ and $h_{\mathrm{ac}}$, respectively.

matrix through the ion incidence angle $\theta$, which reads

$$
R(\theta)=\left(\begin{array}{rr}
\cos \theta & -\sin \theta \\
\sin \theta & \cos \theta
\end{array}\right) .
$$

Thus, in laboratory coordinates, $\vec{\nabla} \cdot \tau=\left(\partial_{z^{\prime}} \tau_{z z} \sin \theta,-\right.$ $\left.\partial_{z^{\prime}} \tau_{z z} \cos \theta\right)$.

Using the above relations, the continuity condition, Eq. (2), and the Navier-Stokes equation, Eq. (3), reduce to the following system for $u(x, z, t), w(x, z, t)$, and $p(x, z, t)$ :

$$
\begin{gathered}
\partial_{x} u+\partial_{z} w=0, \\
-\partial_{x} p+\mu\left(2 \partial_{x x}^{2} u+\partial_{z z}^{2} u+\partial_{x z}^{2} w\right)+\partial_{z^{\prime}} \tau_{z z} \sin \theta=0, \\
-\partial_{z} p+\mu\left(2 \partial_{z z}^{2} w+\partial_{x x}^{2} w+\partial_{x z}^{2} u\right)-\partial_{z^{\prime}} \tau_{z z} \cos \theta=0 .
\end{gathered}
$$

From Eqs. (9) and (10), we observe that the cumulative effect of the ion damage in the bulk enters through the gradient of the stress tensor along the ion beam trajectory, and depends only on the distance to the surface. It is difficult to obtain analytical results for a general functional form of this gradient, while current MD data do not allow to determine it accurately either. However, we can approximate such a derivative as a finite difference between the amorphous-crystalline (a-c), $h_{\mathrm{ac}}$, and the free, $h$, interfaces, namely,

$$
\partial_{z^{\prime}} \tau_{z z} \simeq \frac{\tau_{z z}(h)-\tau_{z z}\left(h_{\mathrm{ac}}\right)}{d_{z^{\prime}}},
$$

where $d_{z^{\prime}}$ is the distance between both interfaces along the ion beam trajectory. This distance depends on the average thickness of the damaged amorphous layer, $R_{0}$, and the 
local angle of incidence $\gamma_{0}$, see Fig. 5. Using trigonometry we have that, for small slopes, $d_{z^{\prime}} \approx R_{0} / \cos \left(\gamma_{0}\right)$. The local angle of incidence is $\gamma_{0}=\theta-\gamma$ where $\gamma=\tan ^{-1} \partial_{x} h$ is the angle between the local surface normal and the $z$ axis in the laboratory coordinate system.

Considering now Eqs. (9) and (10), we can rewrite the components of the stress gradient acting in the bulk of the fluid layer as those of an effective body force $\mathbf{b}=\left(b_{x}, b_{z}\right)$, which contains relevant physical information for the layer motion and had been introduced earlier in Ref. [10] on a more phenomenological basis. Under the approximation in Eq. (11), these components reduce to

$$
\begin{gathered}
b_{x}=\frac{\Delta \tau_{z z}}{R_{0}} \cos (\theta-\gamma) \sin \theta, \\
b_{z}=-\frac{\Delta \tau_{z z}}{R_{0}} \cos (\theta-\gamma) \cos \theta,
\end{gathered}
$$

where $\Delta \tau_{z z} \equiv \tau_{z z}(h)-\tau_{z z}\left(h_{\mathrm{ac}}\right)$ denotes the difference in stress between the free and the a-c interfaces.

We now need to supplement Eqs. (8)-(10) with proper boundary conditions [10] at the free surface, $z=h(x, t)$,

$$
\begin{gathered}
\left.\frac{D z}{D t}\right|_{z=h}-w(z=h)=j_{\mathrm{er}}, \\
\hat{\mathbf{n}} \cdot \tau^{F} \cdot \hat{\mathbf{n}}-\sigma \kappa=\hat{\mathbf{n}} \cdot \tau \cdot \hat{\mathbf{n}}, \\
\hat{\mathbf{n}} \cdot \tau^{F} \cdot \hat{\mathbf{t}}=\hat{\mathbf{n}} \cdot \tau \cdot \hat{\mathbf{t}},
\end{gathered}
$$

and at the a-c interface, $z=h_{\mathrm{ac}}(x, t)$,

$$
\begin{gathered}
u\left(z=h_{\mathrm{ac}}\right)=0, \\
\left.\frac{D z}{D t}\right|_{z=h_{\mathrm{ac}}}-w\left(z=h_{\mathrm{ac}}\right)=j_{\mathrm{am}},
\end{gathered}
$$

where $D / D t$ is a convective derivative [51], $\hat{\mathbf{n}}=$ $\left(-\partial_{x} h, 1\right) / \sqrt{1+\left(\partial_{x} h\right)^{2}}$ and $\hat{\mathbf{t}}=\left(1, \partial_{x} h\right) / \sqrt{1+\left(\partial_{x} h\right)^{2}}$ are the unit normal and tangent vectors, respectively, $\sigma$ is surface tension, and $\kappa=\partial_{x x}^{2} h /\left[1+\left(\partial_{x} h\right)^{2}\right]^{3 / 2}$ is the local curvature at the free surface.

The currents $j_{\mathrm{er}}$ and $j_{\mathrm{am}}$ in Eqs. (14) and (18) account for the rates of erosion and amorphization at the free and a-c interfaces, respectively, and depend on the local geometry of the surface, $h$, and the amorphous-crystalline interface, $h_{\mathrm{ac}}$. Considering that the amorphous layer has reached a stationary density and average thickness, and taking into account the observation that purely erosive contributions are less relevant than the transport of matter in order to account for the critical angle for pattern formation, we approximate $j_{\mathrm{er}}=j_{\mathrm{am}}=0$. The ensuing Eqs. (14) and (18) thus become kinetic equations that implement the time evolution of $h(x, t)$ and $h_{\mathrm{ac}}(x, t)$ as being due to the motion of the amorphous layer. In particular, this allows us to neglect the dynamics of the crystalline phase underneath the a-c interface. Beyond this approximation, a simple choice is to set both rates equal to the erosion velocity due to sputtering as in, e.g., the $\mathrm{BH}$ theory $[10,18]$. We will return to this issue later when considering the velocity of inplane propagation for the ripples.

The right-hand sides (rhs) of Eqs. (15) and (16) are the normal and tangential components of the stress created by the ion at the free surface. Consistent with our MD results, the stress tensors in the laboratory and in the ion trajectory coordinate systems are related by

$$
\tau=R(\theta) \tau^{\prime} R(\theta)^{T}=\tau_{z z}(h)\left(\begin{array}{rr}
\cos (2 \theta) & \sin (2 \theta) \\
\sin (2 \theta) & -\cos (2 \theta)
\end{array}\right) .
$$

Equations (8)-(10) are nonlinear and cannot be solved analytically. Nevertheless, they are amenable to standard perturbative methods as the linear stability analysis, whose details are provided in Appendix. The main idea of this analysis is to obtain planar (i.e., $x$-independent) solutions for $p, u, w$, $h$, and $h_{\text {ac }}$, and then study the evolution of small perturbations around them which are periodic in space. The amplitude of these perturbations is exponentially amplified or damps out in time, at a rate $\omega_{q}$ (linear dispersion relation), where $q$ is space wave vector.

Let us note at this point that, beyond the derivation of the body force, Eqs. (12) and (13), which is grounded in results of our present MD simulations, the hydrodynamic model just put forward is not far from previous formulations [11,27]. A next crucial difference with these is the nonflat boundary condition set in our linear stability analysis, whereby the a-c interface is a vertical and horizontal shift of the free interface, as observed in our experiments [see Figs. 2(b) and 3], see Eq. (A14). This provides the a-c interface with a nontrivial role, which is behind a number of the novel results discussed in the next section.

\section{LINEAR STABILITY ANALYSIS RESULTS}

The linear stability analysis in Appendix takes its simplest form by noting that, as derived from the experiments [see again Figs. 2(b) and 3], the thickness of the damaged amorphous layer is much smaller than the ripple wavelength, $R_{0} / \lambda \ll 1$, as in the so-called shallow-water approximation to the flow of thin fluid films [51]. In this limit, and denoting $s \equiv \sin (2 \theta)$ and $c \equiv \cos (2 \theta)$, we finally find that the imaginary and real parts of the linear dispersion relation, $\omega_{q}$, are given by

and

$$
\operatorname{Im}\left(\omega_{q}\right)=\frac{R_{0}\left\{-3\left[\Delta \tau_{z z}+2 \tau_{z z}(0)\right]+\left[5 \Delta \tau_{z z}+12 \tau_{z z}(0)\right] \cos (2 \theta)\right\}}{6 \mu \tan \theta} q+\frac{R_{0}^{2} \sigma}{3 \mu \tan \theta} q^{3}+\mathcal{O}\left(q^{5}\right)
$$

$$
\operatorname{Re}\left(\omega_{q}\right)=-\frac{R_{0}^{2}\left\{3\left[\Delta \tau_{z z}+16 \tau_{z z}(0)\right] s^{2}+16\left[\Delta \tau_{z z}+3 \tau_{z z}(0)\right] c\right\}}{96 \mu \sin ^{2} \theta} q^{2}-\frac{\sigma R_{0}^{3}}{6 \mu \sin ^{2} \theta} q^{4}+\mathcal{O}\left(q^{6}\right)
$$




\section{A. Real part of the dispersion relation: Pattern formation}

In order to have a morphologically unstable system in which a pattern appears with a characteristic long wavelength, a positive real part of the dispersion relation is required for small values of the wave vector $q$. By inspection of Eq. (21) we see that since $\Delta \tau_{z z}=\tau_{z z}(0)-\tau_{z z}\left(h_{\mathrm{ac}}\right)$, this can only occur if the expression $3\left[17 \tau_{z z}(0)-\tau_{z z}\left(h_{\mathrm{ac}}\right)\right] \sin ^{2}(2 \theta)+16\left[4 \tau_{z z}(0)-\right.$ $\left.\tau_{z z}\left(h_{\mathrm{ac}}\right)\right] \cos (2 \theta)$ is negative. In such a case, $\operatorname{Re}\left(\omega_{q}\right)$ reaches its maximum positive value for $q=q_{m}$, where

$$
q_{m}=\sqrt{\frac{3\left[\tau_{z z}\left(h_{\mathrm{ac}}\right)-17 \tau_{z z}(0)\right] s^{2}+16\left[\tau_{z z}\left(h_{\mathrm{ac}}\right)-4 \tau_{z z}(0)\right] c}{32 \sigma R_{0}}} .
$$

We can further characterize the conditions under which ripples form by considering two complementary cases, namely, when $4 \tau_{z z}(0)>\tau_{z z}\left(h_{\mathrm{ac}}\right)$ and when $4 \tau_{z z}(0)<\tau_{z z}\left(h_{\mathrm{ac}}\right)$. Thus we obtain that the system is unstable (pattern-forming) if

$$
\begin{aligned}
\frac{\cos (2 \theta)}{\sin ^{2}(2 \theta)} & <-\frac{3}{16} \frac{17 \tau_{z z}(0)-\tau_{z z}\left(h_{\mathrm{ac}}\right)}{4 \tau_{z z}(0)-\tau_{z z}\left(h_{\mathrm{ac}}\right)} \text { for } \\
4 \tau_{z z}(0) & >\tau_{z z}\left(h_{\mathrm{ac}}\right) \text { (ripple formation for } \theta>\theta_{c} \text { ), }
\end{aligned}
$$

or if

$$
\begin{aligned}
\frac{\cos (2 \theta)}{\sin ^{2}(2 \theta)} & >-\frac{3}{16} \frac{17 \tau_{z z}(0)-\tau_{z z}\left(h_{\mathrm{ac}}\right)}{4 \tau_{z z}(0)-\tau_{z z}\left(h_{\mathrm{ac}}\right)} \text { for } \\
4 \tau_{z z}(0) & \left.<\tau_{z z}\left(h_{\mathrm{ac}}\right) \text { (ripple formation for } \theta<\theta_{c}^{*}\right) .
\end{aligned}
$$

It is useful to note that the function $\cos (2 \theta) / \sin ^{2}(2 \theta)$ is an unbounded decreasing function which becomes negative for $\theta>45^{\circ}$. For fixed values of $\tau_{z z}(0)$ and $\tau_{z z}\left(h_{\mathrm{ac}}\right)$, the system will be pattern-forming for any angle larger than a critical value, $\theta_{c}$, when $4 \tau_{z z}(0)>\tau_{z z}\left(h_{\mathrm{ac}}\right)$ (non-BH scenario), and for any angle which is smaller than a critical angle, $\theta_{c}^{*}$, when $4 \tau_{z z}(0)<\tau_{z z}\left(h_{\mathrm{ac}}\right)$ (BH-like scenario). These critical angles are obtained when the inequalities appearing in (23) and (24) become equalities. We then get

$$
\begin{aligned}
\Theta_{c} & =\frac{1}{2} \cos ^{-1}\left(\frac{-1+\sqrt{1+4 f^{2}}}{2 f}\right) \quad \text { with } \\
f & \equiv-\frac{3(17 g-1)}{16(4 g-1)}
\end{aligned}
$$

where $g$ has been defined as the ratio between the stress values at the free and a-c interfaces, $g \equiv \tau_{z z}(0) / \tau_{z z}\left(h_{\mathrm{ac}}\right)$. The value of the critical angle predicted for each $g$ is depicted in Fig. 6, according to Eq. (25). In terms of this parameter, $g<1 / 4$ leads to the non-BH scenario in which the system undergoes ripple formation for any angle larger than a critical value, $\Theta_{c}(g<1 / 4) \equiv \theta_{c}$. This is the situation expected for the experimental cases discussed here. As implied by the left branch of Fig. 6, the value of this critical angle ranges from $\theta_{c}=0^{\circ}$ up to $\theta_{c}=61.8^{\circ}$. Meanwhile, the BH-like scenario applies if $g>1 / 4$, namely, the system is pattern-forming for angles smaller than a critical value, $\Theta_{c}(g>1 / 4) \equiv \theta_{c}^{*}$, which ranges in turn between $61.8^{\circ}$ and $90^{\circ}$, see the right branch of Fig. 6.

It should be also noted that, in the limiting case in which $g=1 / 4$, namely when $4 \tau_{z z}(0)=\tau_{z z}\left(h_{\mathrm{ac}}\right)$, the system will be pattern-forming (respectively, nonpattern forming) for any

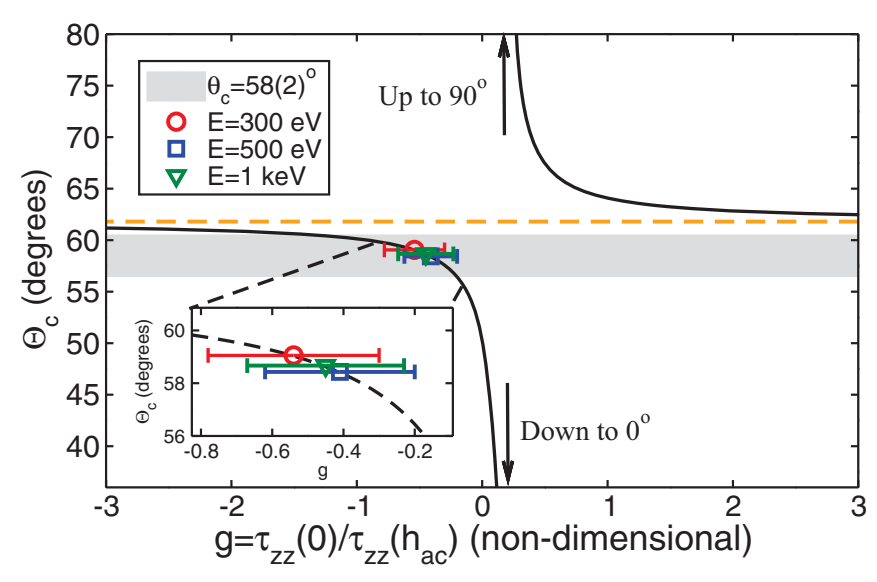

FIG. 6. (Color online) Dependence of the critical angle $\Theta_{c}$ with the stress ratio $g$, Eq. (25). The horizontal gray bar indicates the experimental critical angle range for $\mathrm{Xe}^{+} \rightarrow \mathrm{Si}\left(\theta_{c}=58 \pm 2^{\circ}\right)$. The symbols show values from MD simulations, see legend. The inset is a zoom to show the weak dependence of $\theta_{c}$ with $E$. The orange dashed horizontal line is a guide to the eye with asymptote $\theta=61.8^{\circ}$.

incidence angle if the stress at the surface $\tau_{z z}(0)$ is negative (respectively, positive).

Once stress conditions are such that ripples form, their characteristic wavelength is given by

$$
\lambda=2 \pi / q_{m} .
$$

Hence a power expansion of Eq. (22) close to the type-II transition leads to

$$
\lim _{\theta \rightarrow \theta_{c}} \lambda \sim\left|\theta-\theta_{c}\right|^{-1 / 2},
$$

where the critical angle is to be understood as $\theta_{c}$ or $\theta_{c}^{*}$ depending on the relative values of $\tau_{z z}(0)$ and $\tau_{z z}\left(h_{\mathrm{ac}}\right)$, as just discussed. Note that the value of the critical exponent in Eq. (1) characterizing the divergence of $\lambda$ at the transition is robust to the conditions on stress, and hence universal within the present model. This is not the case for the critical angle. Namely, we obtain that the value of the critical angle for pattern formation depends explicitly on the form of the space distribution of ioninduced stress, as described by parameter $g$ within the present approximations. Such a distribution is expected to change when comparing, e.g., different ion species for fixed target and energy, see Sec. V below. Moreover, our results even allow for a novel pattern forming scenario which differs from the non-BH behavior commonly found under impurity-free conditions on $\mathrm{Si}$. Such BH-like scenario within which ripples form for small incidence angles, including normal incidence, is analogous to the situation under impurity co-deposition or for irradiation of binary targets [17]. This suggests considering the relevance of ion-induced stress in such complex experimental setups.

\section{B. Imaginary part of the dispersion relation: Ripple propagation}

The full linear dispersion relation (A22) still provides further detailed information on the ripple structure, valid within the early-time evolution dominated by the linear instability. Specifically, under pattern-forming conditions, the 
ensuing ripple structure travels coherently across the surface plane [8,9,17], with a velocity $V$ determined by the imaginary part of $\omega_{q}$, Eq. (20) [52,53]. In our case, to lowest order,

$$
\begin{aligned}
V & =-\frac{\operatorname{Im}\left(\omega_{q_{m}}\right)}{q_{m}}=\frac{R_{0}\left\{3\left[3 \tau_{z z}(0)-\tau_{z z}\left(h_{\mathrm{ac}}\right)\right]-\left[17 \tau_{z z}(0)-5 \tau_{z z}\left(h_{\mathrm{ac}}\right)\right] c\right\}}{6 \mu \tan \theta}-\frac{R_{0}^{2} \sigma}{3 \mu \tan \theta} q_{m}^{2} \\
& =\frac{R_{0}^{2}}{96 \mu \tan \theta}\left\{48\left[3 \tau_{z z}(0)-\tau_{z z}\left(h_{\mathrm{ac}}\right)\right]-48\left[7 \tau_{z z}(0)-2 \tau_{z z}\left(h_{\mathrm{ac}}\right)\right] c+3\left[17 \tau_{z z}(0)-\tau_{z z}\left(h_{\mathrm{ac}}\right)\right] s^{2}\right\} \\
& =V_{c} S_{\mathrm{ac}}[-99+339 g+32(4-13 g) \cos (2 \theta)+(3-51 g) \cos (4 \theta)] / \tan \theta,
\end{aligned}
$$

where $V_{c} \equiv R_{0} \tau_{z z}\left(h_{\mathrm{ac}}\right) /(192 \mu)$ is a characteristic velocity scale for the system, and $S_{\mathrm{ac}} \equiv \operatorname{sign}\left[\tau_{z z}\left(h_{\mathrm{ac}}\right)\right]$. Positive velocity values indicate that ripples propagate in the same direction as the ion projection on the surface. We see that the magnitude and sign of the ripple velocity both depend on experimental conditions through parameters $\tau_{z z}(h), \tau_{z z}\left(h_{\mathrm{ac}}\right), R_{0}$, etc. Hence, $V$ is another nonuniversal quantity, in the same sense as $\theta_{c}$ is.

To date, not many experimental reports are available on the velocity of ripple motion and its dependence on experimental conditions. Experiments on Si [54] and glass [55] obtained positive values for the ripple velocity (a fact termed ripple propulsion [55]), opposite to the predictions of the $\mathrm{BH}$ model for the corresponding angles of incidence. Monte Carlo simulations of atomistic models $[8,56]$ similarly contradicted the $\mathrm{BH}$ predictions. This disagreement suggested the relevance of physical mechanisms beyond those considered in the $\mathrm{BH}$ theory for this type of systems [8]. However, more recent experiments on Si are available [57] in which, contrasting with ripple amplification, BH-like erosive effects are argued to be relevant for ripple propagation at non glancing incidence angles. In particular, for increasing $\theta$ the sign of the ripple velocity is seen to change from positive to the negative value expected within a $\mathrm{BH}$ description. We can explore this change in the sign of the ripple velocity within our continuum model. As we are considering only linear effects, we take into account sputtering by simply adding to (28) the BH prediction theory for the ripple velocity, namely $[18,55]$,

$$
V_{\text {sputtering }}=\frac{J}{n}\left[Y(\theta) \sin \theta-\frac{d Y(\theta)}{d \theta} \cos \theta\right],
$$

where $J$ is the ion flux, $n$ is the surface atomic density, and $Y(\theta)$ is the sputtering yield. For the latter, in principle, one can employ the approximate expression deduced by BH from Sigmund's distribution for energy deposition [18]. Here we employ Yamamura's corrected expression for $Y(\theta)$, given by

$$
\begin{aligned}
Y(\theta)= & Y(0) \exp \left[-\frac{a^{2} / \sigma^{2}}{2\left(1+b^{2} \mu^{2} / \sigma^{2}\right)}\right. \\
& \left.+a^{2} / 2 \sigma^{2}-\Sigma \sqrt{1+b^{2}}\right] \sqrt{\frac{1+b^{2}}{1+b^{2} \mu^{2} / \sigma^{2}}} .
\end{aligned}
$$

Here, $b=\tan \theta, \Sigma$ is the Yamamura coefficient, and $a, \mu$, and $\sigma$ are the parameters of Sigmund's Gaussian distribution [58]. Hence the total ripple velocity will be the sum of Eqs. (28) and (29). The relative weight of each contribution will be controlled by the prefactors $V_{c}$ and $J / n$. For the sake of clarity, we introduce a new dimensionless parameter

$$
B \equiv \frac{V_{c}}{J / n}=\frac{R_{0} \tau_{z z}\left(h_{\mathrm{ac}}\right) n}{192 J \mu},
$$

in such a way that, when $B \rightarrow \infty(B \rightarrow 0)$, only the effects of stress (sputtering) are taken into account.

In Fig. 7, we show the predictions of the present ion-induced solid flow theory in combination with the Sigmund-Yamamura prediction for the ripple velocity, for different values of $B$, considering regimes dominated by each one of the two mechanisms considered, stress and sputtering. In that figure, we plot the normalized total velocity,

$$
V_{\text {ripple }}=\frac{V+V_{\text {sputtering }}}{V_{c}}
$$

where $V$ and $V_{\text {sputtering }}$ are given by Eqs. (28) and (29) with $\Sigma=0.046, a / \sigma=2.04$, and $\mu / \sigma=0.66$ (see Ref. [59]). We have used these values as a proof of concept of our ideas although we do not intend to describe quantitatively a specific experimental system at this point. When the ripple propagation is dominated by stress ( $B=1 \mathrm{in} \mathrm{Fig.} \mathrm{7),} \mathrm{the} \mathrm{theory} \mathrm{predicts}$ ripple propulsion, i.e., ripple motion is parallel to the projection of ion beam on the target plane, for all incidence angles above a new critical value, $\hat{\theta}_{c}$, see blue line in Fig. 7. This value is

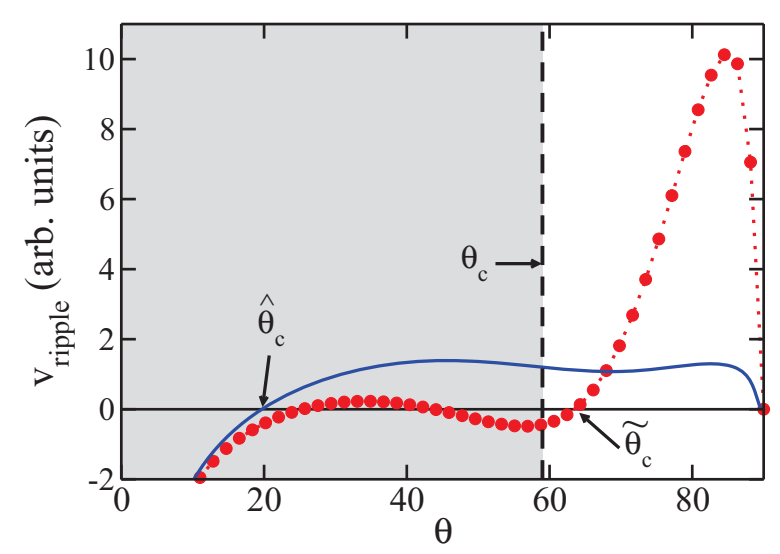

FIG. 7. (Color online) Ripple propagation velocity vs incidence angle under a non-BH scenario for ripple formation with $g=-0.5$ [the value obtained in Fig. 4(a)], $\Sigma=0.046, a / \sigma=2.04$ and $\mu / \sigma=$ 0.66 (see Ref. [59]), for $B=1$ (blue solid line) and $B=0.1$ (red circles). The red dotted line is a guide to the eye. The shaded area is the region where the surface is morphologically stable (there are no ripples), delimited by the critical angle for pattern formation (vertical black dashed line) at $\theta=\theta_{c}$. The values of the transition angles mentioned in the text, $\hat{\theta}_{c}$ and $\tilde{\theta}_{c}$, are indicated. 
independent of $\theta_{c}$ and is located around $20^{\circ}$, hence outside the $\theta$ region in which ripples actually form. Moreover, $\hat{\theta}_{c}$ is robust with respect to the value of $g$ while, as shown in the previous section, $\theta_{c}$ depends strongly on the distribution of ion-induced stress, possibly as in experiments. Figure 7 also shows a case in which sputtering is the dominant mechanism responsible for ripple propagation ( $B=0.1$ ), see red circles and dotted line in Fig. 7. In this case, using the parameters in Refs. [19,21], we obtain a transition in the sign of the ripple velocity for a value of the incidence angle $\tilde{\theta}_{c}$ around $65^{\circ}-70^{\circ}$, akin to the recent experiments mentioned above [57].

A more detailed theoretical study of ripple propagation can be performed along similar lines, exploring in particular the (to date, unobserved) BH-like scenario for pattern onset that has been discussed in the previous section, and has been submitted elsewhere [60]. While in the next section we restrict experimental validation of our present model to ripple formation, systematic assessment of predictions on ripple propagation seems interesting indeed, and will be the subject of future work.

\section{COMPARISON WITH EXPERIMENTS AND DISCUSSION}

The results presented in the preceding sections allow to understand the experimental observations in Table I within an unified framework. Thus our MD results for $\mathrm{Xe}^{+}$yield different signs for the ion-induced stress at the free and a-c interfaces, namely, $\tau_{z z}(0)>0$ and $\tau_{z z}\left(h_{\mathrm{ac}}\right)<0$, see Fig. 4(a). Hence $g<0$, and a non-BH scenario takes place, with $\theta_{c}<$ $61.8^{\circ}$. Interestingly, for the case of Si targets, we are aware of no reported $\theta_{c}$ value to date which exceeds $62^{\circ}$. From Table I, heavier ions induce higher values of $\theta_{c}$, suggesting a larger value of the ion-induced surface stress $\tau_{z z}(0)$, as compared to the stress at the a-c interface $\tau_{z z}\left(h_{\mathrm{ac}}\right)$. Our MD values for stress imply a value of $\theta_{c}$ which is compatible (within error bars) with our experimental result, see Fig. 6. As previously observed [11,19,21], $\theta_{c}$ depends weakly with $E$, see the figure inset.

In Fig. 8(a), we show the experimental $\theta$-dependence of the ripple wavelength for $\theta>\theta_{c}$ in the case of $\mathrm{Ar}^{+}$(adapted from Ref. [11]) and $\mathrm{Xe}^{+}$ions, and compare it with the

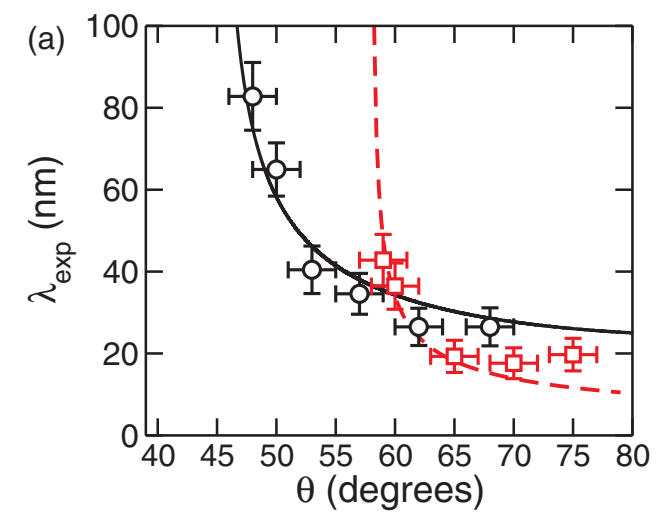

predictions from Eq. (26). Indeed, $\theta_{c}^{\mathrm{Xe}} \simeq 58^{\circ}>\theta_{c}^{\mathrm{Ar}^{+}} \simeq 46^{\circ}$, the nonrippled phase being more dominant for $\mathrm{Xe}^{+}$, as reported in Refs. [11,20,21,28], hence the value of the critical angle is nonuniversal with respect to ion species. In contrast, Fig. 8(a) confirms the independence with respect to the ion species (universality) of the exponent value $1 / 2$, which characterizes the divergence of $\lambda$ for $\theta \rightarrow \theta_{c}$.

Regarding the role of the ion energy in the pattern properties, note that Eqs. (22) and (26) allow to infer the dependence of $\lambda$ with physical parameters other than the incidence angle, and in particular with $E$. Assuming $E$ independent surface tension $\sigma$, using MD data from Fig. 4(b) for $\mathrm{Xe}^{+}$, and similarly to the case of $\mathrm{Ar}^{+}$[11], linear scaling $\lambda \sim E$ is approximately obtained, in good agreement with our experimental observations, see Fig. 8(b). While the classic BH theory with thermal surface diffusion leads to a decrease of $\lambda$ with $E$ [8], a detailed account of the dependence of collision cascades with energy does allow for an increasing wavelength in the form $\lambda \sim E^{p}$ for appropriate values of $p>0$, still within an essentially sputtering-dominated view of the IBS process $[61,62]$. However, such a refinement does not avoid the failure of erosive approaches to predict $\theta_{c} \neq 0$, hence the experimental increase of the ripple wavelength with energy requires justification beyond the $\mathrm{BH}$ paradigm. Our hydrodynamic model predicts approximately linear scaling for the case of $\mathrm{Si}$, as observed, even if the theoretical prefactor overestimates the experimental value. Interestingly, close-tolinear scaling has also been reported for other amorphizable targets like $\mathrm{SiO}_{2}$ [63], graphite [62], or amorphous carbon [64]. This suggests the generality of viscous flow relaxation of ion-induced stress as a mechanism for ripple formation also in this class of systems.

It is uncertain whether other materials that also lead to nanopatterning under IBS can be accounted for along similar lines, especially metals. The main difference is that for metals no proper amorphous layer forms under low energy IBS [8,32], so that material transport at the surface is dominated by diffusion, rather than by viscous flow. For this class of systems, we expect reaction-diffusion models (specifically, the so-called two-field models in this context $[9,13,17])$ to be more indicated, whereby the evolution of the surface height is coupled to that of the concentration field of diffusing

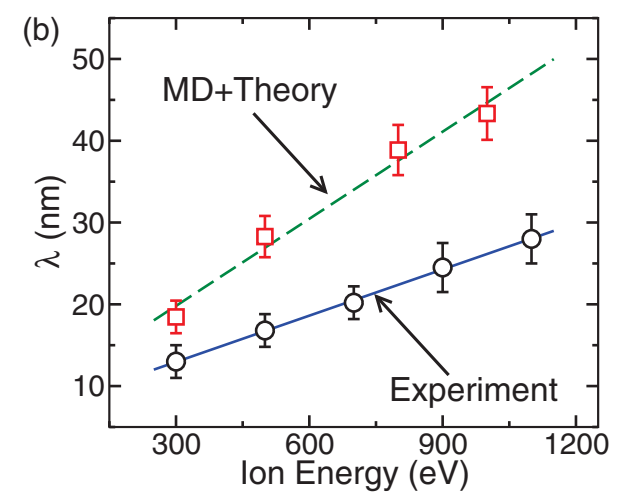

FIG. 8. (Color online) (a) Experimental ripple wavelength vs $\theta$ for $\mathrm{Si}$ at $500 \mathrm{eV}$, using $\mathrm{Ar}^{+}$(black circles, from $\mathrm{Ref}^{\text {[11]) and Xe }}{ }^{+}$(red squares) ions. The solid black and the dashed red lines are fits to Eq. (27) for $\theta_{c}=46^{\circ}$ and $58^{\circ}$, respectively. (b) $\lambda$ vs $E$ for Xe ${ }^{+}$according to Eq. (26) using MD data (red squares) and experiments (black circles). Straight lines are linear fits. 
species. Actually, this type of models have recently shown quantitative agreement with IBS nanopatterning experiments on, e.g., gold surfaces [65,66]. Experience with analogous (albeit macroscopic) ripple formation systems like Aeolian or underwater sand dunes suggests that two-field models can provide good shallow-water approximations of full-fledge hydrodynamic systems [67]. Hence suitable extensions of our present hydrodynamic model to encompass metallic systems might be envisaged.

\section{CONCLUSIONS}

In summary, we have shown that the combination of MD simulations for the microscopic stress generation with a hydrodynamic theory accounting for its mesoscopic relaxation provides a robust framework that can explain the experimental variability of IBS nanopatterning of $\mathrm{Si}$, and possibly other important targets that become amorphous under the present type of irradiation, typically semiconductors.

Our approach can predict the values of quantities that characterize the ripple formation process, like the critical angle, the divergence of the ripple wavelength at the type-II transition, and the scaling of the ripple wavelength with energy, including their universality or lack thereof with respect to, e.g., a change in the ion species. We obtain good agreement with our own experiments with $\mathrm{Xe}^{+}$and $\mathrm{Ar}^{+}$ions, as well as with other as summarized in Table I. Our predictions include even the maximum value of the critical angle that can take place within a non-BH scenario for ripple formation, appropriate for $\mathrm{Si}$. As improvements in our approach over previous recent models, we can stress the experimental assessment and continuum description of the corrugation of the a-c interface, which has a nontrivial effect in the pattern formation and dynamics.

Nevertheless, unavoidably our modeling requires approximations that are related, on the one hand, with computational feasibility in connection to MD simulations; on the other hand, with analytical tractability in the case of the continuum description. Relaxation of such approximations may possibly affect, e.g., the numerical values obtained for a number model predictions, like the $61.8^{\circ}$ upper bound for $\theta_{c}$ under non-BH scenarios, the value of the ripple in-plane velocity, etc. In the process of improving upon our current approximations, it would be interesting to make contact with kinetic descriptions like rate equations for flow defect dynamics [50], which have been recently validated against stress measurements on $\mathrm{Si}$ [42]. Still, we believe that our general approach and conclusions can apply to a wide range of systems and experimental conditions. Thus one can consider the effect on the value of $\theta_{c}$ of experimental parameters other than ion/target combination through changes in the space distribution of stress, and thus identify similarities and differences among different cases. Our results also allow for the possibility of critical angles smaller than $45^{\circ}$, as, e.g., for silica [68], which have not been reported too frequently, and even suggest a potential role for stress in the BH-like scenarios occurring in the irradiation of binary compounds or in the presence of impurities. We have also found that both, the velocity and the direction of ripple propagation can be affected by the incidence angle, as recently observed in experiments [57]. It would be interesting to compare our results with similar experiments performed for other ion species, in order to assess the influence of the stress distribution on the in-plane propagation of the pattern. All these aspects seem to deserve future attention, with the ultimate goal to reach full understanding of the basic physical principles underlying this fascinating route to surface nanostructuring.

\section{ACKNOWLEDGMENTS}

This work has been partially supported by MICINN (Spain) Grant MAT2011-13333-E, and MINECO (Spain) Grants FIS2012-38866-C05-01, FIS2012-38866-C05-05, FIS201347949-C2-2-P and FIS2012-32349. TEM work has been conducted at LABMET laboratory, associated with Red de Laboratorios of CAM, Spain. A.M.-B. acknowledges support from MINECO, through FPI scolarship BES-2010-036179. A.R.C. acknowledges funding from Juan de la Cierva program (Spain) under Contract No. JCI-2012-14509. We thank H. M. Urbassek and C. Anders for help with the MD simulations, and M. Plapp for discussions.

\section{APPENDIX: LINEAR STABILITY ANALYSIS}

\section{Flat solution}

In order to perform a linear stability analysis of the problem, we first need to obtain its flat (i.e., $x$-independent) steadystate solution. This will provide a reference (zeroth order) configuration, whose stability under periodic (first order) perturbations will be later assessed. For such a flat solution, the profiles of the steady pressure, $p_{0}(z)$, and horizontal, $u_{0}(z)$, and vertical, $w_{0}(z)$, velocities will be functions of the depth $z$ only; their shapes are governed by the reduced form of Eqs. (8)-(10), which read

$$
\begin{gathered}
\partial_{z} w_{0}=0 \\
\mu \partial_{z z}^{2} u_{0}+\frac{\Delta \tau_{z z}}{R_{0}} \cos \theta \sin \theta=0 \\
-\partial_{z} p_{0}+2 \mu \partial_{z z}^{2} w_{0}-\frac{\Delta \tau_{z z}}{R_{0}} \cos ^{2} \theta=0
\end{gathered}
$$

If we consider an inertial frame of reference in which the planar free interface $h$ is located at $z=0$ while the a-c interface $h_{\mathrm{ac}}$ is located at $z=-R_{0}$, the boundary conditions Eqs. (15)-(18) reduce to

$$
\begin{gathered}
-p_{0}(0)+2 \mu \partial_{z} w_{0}(0)=-\tau_{z z}(0) \cos (2 \theta) \\
\mu \partial_{z} u_{0}(0)=\tau_{z z}(0) \sin (2 \theta) \\
u_{0}\left(-R_{0}\right)=0 \\
w_{0}\left(-R_{0}\right)=0
\end{gathered}
$$

We can easily integrate Eqs. (A1)-(A3) using Eqs. (A4)-(A7) to obtain the steady, planar profiles. From the incompressibility condition, Eq. (A1), and using (A7), we obtain that to this order of approximation there is no vertical component of the flow, $w_{0}(z)=0$. The pressure and the horizontal flow velocity take 
the form

$$
\begin{aligned}
p_{0}(z)=-\frac{\Delta \tau_{z z}}{R_{0}} z \cos ^{2} \theta+\tau_{z z}(0) \cos (2 \theta) \\
u_{0}(z)=\left(-\frac{\Delta \tau_{z z}}{2 R_{0}} z^{2}+2 \tau_{z z}(0) z+\frac{\Delta \tau_{z z} R_{0}}{2}+2 \tau_{z z}(0) R_{0}\right) \\
\times \frac{\sin (2 \theta)}{2 \mu}
\end{aligned}
$$

where we have used that the integral of the stress gradient across the amorphous layer only depends on its difference, $\Delta \tau_{z z}$, between the free and the a-c interfaces, namely, we are considering a constant body force. Equations (A8) and (A9) imply a linear profile for the pressure and a parabolic profile for the horizontal velocity, which reduce to a constant pressure and to a linear horizontal velocity profile, respectively, when $\Delta \tau_{z z}=0$ as in Ref. [27]. In such a case, at the free surface $(z=0)$ the pressure only depends on the stress created by the ions there, $\tau_{z z}(0)$, being maximum for $\theta=0^{\circ}$. For larger angles of incidence the pressure decreases monotonically, being negative for $\theta>45^{\circ}$. On the other hand, the flow velocity at the free surface is minimum at normal and at grazing incidence, and maximum for $\theta=45^{\circ}$.

\section{First-order solution and linear dispersion relation}

We can study how the stability of this planar solution is altered by adding a small perturbation which is periodic in the substrate coordinate $x$ with wave vector $q$. Up to first order in a small parameter $\epsilon$, the pressure, horizontal and vertical velocities, and the free and a-c interfaces take the form

$$
\begin{gathered}
p=p_{0}(z)+\epsilon p_{1}(z) \mathrm{e}^{\omega_{q} t+i q x}, \\
u=u_{0}(z)+\epsilon u_{1}(z) \mathrm{e}^{\omega_{q} t+i q x}, \\
w=w_{0}(z)+\epsilon w_{1}(z) \mathrm{e}^{\omega_{q} t+i q x}, \\
h=\epsilon h_{1} \mathrm{e}^{\omega_{q} t+i q x}, \\
h_{\mathrm{ac}}=-R_{0}+\epsilon h_{1} \mathrm{e}^{\omega_{q} t+i q\left(x-R_{0} \tan \theta\right)},
\end{gathered}
$$

where $\omega_{q}$ is the amplification rate (linear dispersion relation) for the amplitude of the perturbation. It should be noted that we have simply considered that the a-c interface is vertically and horizontally displaced with respect to $h$ by the amounts $-R_{0}$ and $-R_{0} \tan \theta$, respectively, as observed in our experiments [see Figs. 2(b) and 3]. A more elaborate relation between both interfaces can be written; however, Eq. (A14) is enough up to the present order of approximation. As compared with previous works [10,27], it already endows the a-c interface with a nontrivial form and dynamics.
Substituting expressions (A10)-(A14) into Eqs. (8)(10) and expanding $\cos (\theta-\gamma) \approx \cos \theta+\gamma \sin \theta \approx \cos \theta+$ $\left(\partial_{x} h\right) \sin \theta$, we obtain, to first order in $\epsilon$,

$$
\begin{gathered}
i q u_{1}+\partial_{z} w_{1}=0, \\
-i q p_{1}+\mu\left(-2 q^{2} u_{1}+i q \partial_{z} w_{1}+\partial_{z}^{2} u_{1}\right) \\
+i q \frac{\Delta \tau_{z z} h_{1}}{R_{0}} \sin ^{2} \theta=0, \\
-\partial_{z} p_{1}+\mu\left(2 \partial_{z}^{2} w_{1}+i q \partial_{z} u_{1}-q^{2} w_{1}\right) \\
-i q \frac{\Delta \tau_{z z} h_{1}}{2 R_{0}} \sin (2 \theta)=0 .
\end{gathered}
$$

Working similarly with the boundary conditions at the top interface $(z=h)$, we obtain

$$
\begin{gathered}
p_{1}(0)-h_{1} q^{2} \sigma-2 \mu \partial_{z} w_{1}(0)-\frac{\Delta \tau_{z z} h_{1}}{R_{0}} \cos ^{2} \theta=0 \\
\mu\left[i q w_{1}(0)+\partial_{z} u_{1}(0)\right]+i q 2 \tau_{s z z}(0) h_{0} \cos (2 \theta) \\
-\frac{\Delta \tau_{z z} h_{1}}{2 R_{0}} \sin (2 \theta)=0
\end{gathered}
$$

while those at the bottom interface $\left(z=h_{\mathrm{ac}}\right)$ [Eqs. (17) and (18)] lead to

$$
\begin{gathered}
u_{0}^{\prime}\left(-R_{0}\right)+u_{1}\left(-R_{0}\right)=\frac{\Delta \tau_{z z}+2 \tau_{z z}(0)}{2 \mu} h_{1} \sin (2 \theta) \mathrm{e}^{-i q R_{0} \tan \theta} \\
+u_{1}\left(-R_{0}\right)=0, \\
\mathrm{e}^{-i q R_{0} \tan \theta} h_{1} \omega-w_{1}\left(-R_{0}\right)=0 .
\end{gathered}
$$

From Eq. (A15), we can express $u_{1}$ through the first derivative of $w_{1}$ and substitute it into Eq. (A16) to get an expression of $p_{1}$ as a function of $w_{1}$ and its derivatives. If we now write down Eq. (A17) as a function of $w_{1}$, we get a fourth-order equation in terms of derivatives of $w_{1}$ only. Such an equation can be solved using the boundary conditions, Eqs. (A18)-(A21).

Expanding Eq. (14) to first order in $\epsilon$, we get an expression for the linear dispersion relation $\omega_{q}$ as a function of $u_{0}$ and $w_{1}$ at $z=0$, which reads

$$
\omega_{q}=-i q u_{0}(0)+w_{1}(0) .
$$

This result can be simplified further by assuming that the thickness of the damaged amorphous layer, $R_{0}$, is much smaller than the ripple wavelength, $\lambda$, and performing a power expansion in wave-vector $q$. This finally leads to Eqs. (20) and (21) in the main text. Note, within the present description, ripples form if there exist wave-vector values for which the real part of the dispersion relation, $\operatorname{Re}\left(\omega_{q}\right)$, takes positive values. In such a case, the ripple wavelength $\lambda=2 \pi / q_{m}$, where $q_{m}$ is the value for which $\operatorname{Re}\left(\omega_{q}\right)$ reaches its (positive) maximum.
[1] M. Tang and A. Karma, Phys. Rev. Lett. 108, 265701 (2012).

[2] J.-N. Aqua, A. Gouyé, A. Ronda, T. Frisch, and I. Berbezier, Phys. Rev. Lett. 110, 096101 (2013).

[3] P. Kim, M. Abkarian, and H. A. Stone, Nat. Mater. 10, 952 (2011).

[4] B. Andreotti, A. Fourriere, F. Ould-Kaddour, B. Murray, and P. Claudin, Nature (London) 457, 1120 (2009).
[5] D. J. Jerolmack, R. C. Ewing, F. Falcini, R. L. Martin, C. Masteller, C. Phillips, M. D. Reitz, and I. Buynevich, Nat. Geosci. 5, 206 (2012).

[6] H. Trinkaus and A. I. Ryazanov, Phys. Rev. Lett. 74, 5072 (1995).

[7] S. Facsko, T. Dekorsy, C. Koerdt, C. Trappe, H. Kurz, A. Vogt, and H. L. Hartnagel, Science 285, 1551 (1999). 
[8] W. L. Chan and E. Chason, J. Appl. Phys. 101, 121301 (2007).

[9] J. Muñoz-García, L. Vázquez, R. Cuerno, J. A. Sánchez-García, M. Castro, and R. Gago, in Toward Functional Nanomaterials, edited by Z. Wang (Springer, New York, 2009).

[10] M. Castro and R. Cuerno, Appl. Surf. Sci. 258, 4171 (2012).

[11] M. Castro, R. Gago, L. Vázquez, J. Muñoz-García, and R. Cuerno, Phys. Rev. B 86, 214107 (2012).

[12] H. Gnaser, Low Energy Ion Irradiation of Solid Surfaces (Springer-Verlag, New York, 1999).

[13] R. Cuerno, M. Castro, J. Muñoz-García, R. Gago, and L. Vázquez, Nucl. Instr. Meth. Phys. Res. B 269, 894 (2011).

[14] B. Fazio, C. D’Andrea, F. Bonaccorso, A. Irrera, G. Calogero, C. Vasi, P. Gucciardi, M. Allegrini, A. Toma, D. Chiappe et al., ACS Nano 5, 5945 (2011).

[15] S. A. Norris, J. Samela, L. Bukonte, M. Backman, F. Djurabekova, K. Nordlund, C. S. Madi, M. P. Brenner, and M. J. Aziz, Nat. Commun. 2, 276 (2011).

[16] G. Lu and E. Kaxiras, in Handbook of Theoretical and Computational Nanotechnology, edited by M. Rieth and W. Schommers (American Scientific Publishers, Valencia, CA, 2005).

[17] J. Muñoz-García, L. Vázquez, M. Castro, R. Gago, A. RedondoCubero, A. Moreno-Barrado, and R. Cuerno, Mater. Sci. Eng., R 86, 1 (2014).

[18] R. Bradley and J. Harper, J. Vac. Sci. Technol. A 6, 2390 (1988).

[19] C. S. Madi, B. Davidovitch, H. B. George, S. A. Norris, M. P. Brenner, and M. J. Aziz, Phys. Rev. Lett. 101, 246102 (2008).

[20] C. S. Madi, E. Anzenberg, K. F. Ludwig, Jr., and M. J. Aziz, Phys. Rev. Lett. 106, 066101 (2011).

[21] C. S. Madi, H. B. George, and M. J. Aziz, J. Phys.: Condens Matter 21, 224010 (2009).

[22] M. Cross and H. Greenside, Pattern Formation and Dynamics in Nonequilibrium Systems (Cambridge University Press, Cambridge, UK, 2009).

[23] R. M. Bradley and P. D. Shipman, Phys. Rev. Lett. 105, 145501 (2010).

[24] R. M. Bradley, Phys. Rev. B 87, 205408 (2013).

[25] M. Z. Hossain, K. Das, J. B. Freund, and H. T. Johnson, Appl. Phys. Lett. 99, 151913 (2011).

[26] S. A. Norris, Phys. Rev. B 85, 155325 (2012).

[27] S. A. Norris, Phys. Rev. B 86, 235405 (2012).

[28] H. Hofsäss, O. Bobes, and K. Zhang, AIP Conf. Proc. 1525, 386 (2013).

[29] S. Macko, F. Frost, B. Ziberi, D. F. Förster, and T. Michely, Nanotechnol. 21, 085301 (2010).

[30] E. Anzenberg, J. C. Perkinson, C. S. Madi, M. J. Aziz, and K. F. Ludwig, Jr, Phys. Rev. B 86, 245412 (2012).

[31] M. Teichmann, J. Lorbeer, B. Ziberi, F. Frost, and B. Rauschenbach, New J. Phys. 15, 103029 (2013).

[32] U. Valbusa, C. Boragno, and F. B. de Mongeot, J. Phys.: Condens. Matter 14, 8153 (2002).

[33] H. Hofsäss, Appl. Phys. A 114, 401 (2014).

[34] M. L. Nietiadi and H. M. Urbassek, Appl. Phys. Lett. 103, 113108 (2013).

[35] J. C. Perkinson, E. Anzenberg, M. J. Aziz, and K. F. Ludwig, Jr., Phys. Rev. B 89, 115433 (2014).

[36] M. P. Harrison and R. M. Bradley, Phys. Rev. B 89, 245401 (2014).
[37] T. K. Chini, F. Okuyama, M. Tanemura, and K. Nordlund, Phys. Rev. B 67, 205403 (2003).

[38] N. Kalyanasundaram, M. C. Moore, J. B. Freund, and H. T. Johnson, Acta Mater. 54, 483 (2006).

[39] N. Medhekar, W. Chan, V. Shenoy, and E. Chason, J. Phys.: Condens. Matter 21, 224021 (2009).

[40] E. Chason and V. Shenoy, Nucl. Instrum. Methods. Phys. Res. Sec. B 272, 178 (2012).

[41] C. A. Davis, Thin Solid Films 226, 30 (1993).

[42] Y. Ishii, C. S. Madi, M. J. Aziz, and E. Chason, J. Mater. Res. 29, 2942 (2014).

[43] S. Plimpton, J. Comput. Phys. 117, 1 (1995).

[44] J. Tersoff, Phys. Rev. B 38, 9902 (1988).

[45] J. Tersoff, Phys. Rev. B 41, 3248 (1990).

[46] J. Nord, K. Albe, P. Erhart, and K. Nordlund, J. Phys.: Condens. Matter 15, 5649 (2003).

[47] J. F. Ziegler and J. P. Biersack, The Stopping and Range of Ions in Matter (Springer, New York, 1985).

[48] Y. Yin, D. McKenzie, and M. Bilek, Surf. Coat. Technol. 198, 156 (2005).

[49] B. Abendroth, H. Jäger, W. Möller, and M. Bilek, Appl. Phys. Lett. 90, 181910 (2007).

[50] T. M. Mayer, E. Chason, and A. J. Howard, J. Appl. Phys. 76, 1633 (1994).

[51] A. Oron, S. H. Davis, and S. G. Bankoff, Rev. Mod. Phys. 69, 931 (1997).

[52] R. M. M. Mattheij, S. W. Rienstra, and J. H. P. ten Thije Boonkkamp, Partial Differential Equations: Modeling, Analysis, Computation (SIAM, Philadelphia, 2005).

[53] J. Muñoz-García, R. Cuerno, and M. Castro, Phys. Rev. B 78, 205408 (2008).

[54] S. Habenicht, K. P. Lieb, J. Koch, and A. D. Wieck, Phys. Rev. B 65, 115327 (2002).

[55] P. F. A. Alkemade, Phys. Rev. Lett. 96, 107602 (2006).

[56] I. Koponen, M. Hautala, and O.-P. Sievänen, Phys. Rev. Lett. 78, 2612 (1997).

[57] H. Hofsäss, K. Zhang, H. G. Gehrke, and C. Brüsewitz, Phys. Rev. B 88, 075426 (2013).

[58] B. Davidovitch, M. J. Aziz, and M. P. Brenner, Phys. Rev. B 76, 205420 (2007).

[59] Y. Yamamura, C. Mössner, and H. Oechsner, Radiat. Eff. 103, 25 (1987).

[60] A. Moreno-Barrado, M. Castro, J. Muñoz-García, and R. Cuerno (unpublished).

[61] S. Habenicht, W. Bolse, H. Feldermann, U. Geyer, H. Hofsäss, K. Lieb, and F. Roccaforte, Europhys. Lett. 50, 209 (2000).

[62] S. Habenicht, Phys. Rev. B 63, 125419 (2001).

[63] C. C. Umbach, R. L. Headrick, and K.-C. Chang, Phys. Rev. Lett. 87, 246104 (2001).

[64] O. Bobes, K. Zhang, and H. Hofsäss, Phys. Rev. B 86, 235414 (2012).

[65] J. Kim, N. Ha, J. Kim, M. Joe, K. Lee, and R. Cuerno, Nanotechnol. 22, 285301 (2011).

[66] J.-H. Kim, J.-S. Kim, J. Muñoz-García, and R. Cuerno, Phys. Rev. B 87, 085438 (2013).

[67] I. S. Aranson and L. S. Tsimring, Rev. Mod. Phys. 78, 641 (2006).

[68] A. Keller, S. Facsko, and W. Möller, J. Phys.: Cond. Matter 21, 495305 (2009). 